\title{
Left posteroventral pallidotomy results in a deficit in verbal memory
}

\author{
S.F. Crowe ${ }^{\mathrm{a}, *}$, J.D. O’Sullivan ${ }^{\mathrm{b}, \mathrm{c}}$, R.F. Peppard ${ }^{\mathrm{b}}$, \\ P.M. McNeill ${ }^{\text {b }}$, F. Bardenhagen ${ }^{\text {b,d }}$ and \\ S. Bowden ${ }^{\mathrm{b}, \mathrm{d}}$

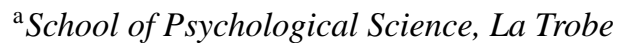 \\ University, Bundoora, Vic., Australia \\ ${ }^{\mathrm{b}}$ Department of Clinical Neuroscience, St Vincent's \\ Hospital, Fitzroy, Vic., Australia \\ ${ }^{\mathrm{c}}$ Department of Neurology, Austin and Repatriation \\ Medical Centre, Melbourne, Vic., Australia \\ ${ }^{\mathrm{d}}$ School of Behavioural Sciences, University of \\ Melbourne, Vic., Australia
}

\begin{abstract}
Whilst pallidotomy is emerging as a popular approach to the treatment to Parkinson's disease, little is yet known about the cognitive effects of this procedure. This study presents 19 patients $(6$ right, 13 left) who were assessed both before and after the procedure on a battery of cognitive tests. The results indicate that subjects with left-sided lesions display significant decline in verbal memory between one and three months following the procedure. The results are consistent with the notion of either a classic amnesic syndrome or a deficit in striato-frontal working memory.
\end{abstract}

Keywords: Pallidotomy, memory, Parkinson's disease, globus pallidus interna, basal ganglia

\section{Introduction}

Lesioning of the globus pallidus is emerging as an important treatment for some aspects of Parkinson's disease (PD). A recent report [9], which indicated improvement in all of the cardinal symptoms of PD and levodopa induced dyskinesias after unilateral posteroventral pallidotomy (PVP), has led to many centres throughout the world adopting the procedure as part of the spectrum of treatment for the disorder. Despite the popularity of PVP, there is as yet little data concerning the effect of the procedure on cognitive functioning.

Riordan et al. [16] note that subjects with a left pallidotomy showed declines in verbal memory, ver-

${ }^{*}$ Corresponding author: Simon F. Crowe, School of Psychological Science, La Trobe University, Bundoora, Vic. 3083, Australia. Tel.: +61 39479 1380; Fax: +61 39479 1829; E-mail: s.crowe@latrobe.edu.au. bal fluency and cognitive flexibility post-operatively, while lesions of the right globus pallidus resulted in enhanced performance on non-verbal memory measures. Lang et al. [10] have noted a similar result to Riordian et al. [16] and have observed that their patients with left sided lesions indicated transient impairments (up to 6 months) on tests of phonemic fluency and on serial list learning, as well as more chronic impairments in category fluency after 12 months.

Riordian et al. [16] proposed that the mechanism of this effect may be due to the fact that lesioning the globus pallidus interna (GPi) may disrupt the subthalamopallidal pathways which results in the disinhibition of pallidal activity. As a second effect the lesion may also disrupt larger frontosubcortical circuits needed for processing information. Taylor and Saint Cyr [19] have proposed a functional system incorporating the association cortex, the caudate nuclei, the pallidum and the substantia nigra, the lateral and dorsal medial nuclei of the thalamus and the dorso-lateral pre-frontal cortex as the substrate of the executive dysfunction noted in Parkinson's disease.

Baron and his group [2, 3] have noted that in their cognitive assessment of 12 patients ( 8 left, 4 right) that only four of their 25 neuropsychological variables changed as a consequence of surgery. These included digit span reversed, the Memory and the Initiation and Perseveration subscales of the Dementia Rating Scale and the Long Delay Memory Discrimination Index of the California Verbal Learning Test. They noted that after they had excluded the data from two subjects who had had post-operative frontal sub-dural haematomas, none of the variables remained significant. No differences were noted as a function of side.

Cullum et al. [4] have observed that of the 19 patients they studied, unilateral pallidotomy (14 right-sided, 5 left-sided) resulted in improvements in verbal learning, non-verbal memory and figural fluency in a majority of patients irrespective of the side of surgery. Soukup et al. [17] also failed to note any deterioration in function following the procedure, however it was not possible to determine if there was a specific effect of side of operation due to the fact that they pooled the data from all of their subjects. 
It seems that there is some diversity of opinion regarding whether deficits in neuropsychological function occur following the procedure and if so on what aspects of cognitive function they impinge. Whilst the role of the striatum in the formation of implicit or procedural memories is well established [18], impairments of explicit working memory have recently been reported in a variety of patients with disease of the striatal system (e.g., [15]). It was the aim of the investigation to attempt to clarify this issue.

\section{Method}

\subsection{Sample}

Nineteen patients underwent unilateral PVP (6 right, 13 left) at St Vincent's Hospital, Melbourne, Australia. Their mean age was 63.6 y (56-77). All patients were right hand dominant and were responsive to levodopa but had persisting symptoms and motor fluctuations despite optimal pharmacotherapy. Patients had a mean duration of symptoms of $11.7 \mathrm{y}$ (4-43). No measure of severity, motor improvement as a consequence of the procedure or demographic data differed as a function of side (all $p>0.05$ ) with the single exception of mean Hoehn and Yahr severity index off medication before operation (Right side 3.8, Left side 2.8, $F(1,17)=$ 5.54, $p=0.03$ ), which indicates that the subjects who received a right sided lesion were more impaired in terms of their motor function before the procedure.

\subsection{Procedure}

The surgery involved a unilateral PVP contralateral to the more disabling symptoms. Preoperatively, the target was identified by MRI localisation, using initial targets based on those described by Laitinen et al. [9] The subject was maintained in a stereotactic frame and the procedure carried out through a frontal burr hole on the coronal suture $2 \mathrm{~cm}$ lateral to the midline. In the majority of cases, semi microelectrode recording was performed and the characteristic neuronal firing pattern within the Globus pallidum interna and externa, the border zone between these two segments and the optic tract was used to determine the dorsal and ventral margins of the GPi along a given trajectory. Electrical macrostimulation (pulse width $0.2 \mathrm{~ms}, 0-10 \mathrm{~V}$ at 5,50 and $100 \mathrm{~Hz}$ ) was undertaken to determine the proximity of the probe relative to the internal capsule and the optic tract. One to five trajectories were tested for each patient. Test lesions were induced at $70^{\circ} \mathrm{C}$ for $20 \mathrm{sec}-$ onds. The permanent lesions were made at $80^{\circ} \mathrm{C}$ for 60 seconds and were performed at $2 \mathrm{~mm}$ levels from the final target up to $8 \mathrm{~mm}$ dorsal along the optimal trajectory to produce a cylindrical lesion. Speech, vision and motor tests were performed throughout the procedure and the visual fields were tested post-operatively.

Clinical assessments based on the Core Assessment Program for Intracerebral Transplantation protocol [11] were performed both 'off' (in the morning 12 hours off medication) and 'on' (best on state after standard levodopa dose) before and one to three months after surgery. These assessments included the Unified Parkinson's Disease Rating Scale, Hoehn and Yahr stage, timed tests of motor function and a dyskinesia score. These preliminary results have been previously reported [13].

Presurgical neuropsychological evaluations were conducted within a week prior to surgery, and followup evaluations were done from one to three months following the surgery. No differences were noted between the patterns of responses observed between one and three months after the procedure. The neuropsychological tests used included: Vocabulary from the Wechsler Adult Intelligence Scale-Revised, Phonemic and Semantic fluency, the Digit Span subtest from the Wechsler Memory Scale-Revised, auditory and visual reaction time, the Raven's Coloured Progressive Matrices Test, the Beck Depression Inventory and the Rey Auditory Verbal Learning Test (RAVLT). All of these tests are commonly employed and are described in standard neuropsychological test compendia [12]. Parallel forms of the cognitive tests were used wherever these were available (i.e., Phonemic and Semantic Fluency, RAVLT) and the versions were randomised with respect to their use in the pre- or post-operative assessment. The patients were investigated on medication both before and after the surgery.

Results of the clinical neurological assessments [13] demonstrated significant improvements in total and contralateral limb UPDRS motor subscores in the 'off' state and total and contralateral limb dyskinesia scores in these subjects. Post-surgical MRI within one week confirmed that lesions were located in the ventral pallidum and that there were no haemorrhages within the needle tract.

\section{Results}

The neuropsychological data were analysed as raw scores and subjected to matched pairs $t$ tests compar- 
Table 1

Performance of the subjects on the battery of Neuropsychological tests as a function of side of procedure and relative to operation

\begin{tabular}{llccc}
\hline Side & Test & Pre operative & Post operative & $p$ \\
\hline Right & Vocabulary & $45.0(15.5)$ & $45.3(10.8)$ & 0.89 \\
& Phonemic fluency & $34.5(21.4)$ & $35.0(11.3)$ & 0.93 \\
& Semantic fluency & $11.3(5.7)$ & $13.8(1.5)$ & 0.53 \\
& Digit span (total) & $14.3(2.7)$ & $15.7(4.4)$ & 0.48 \\
& Auditory reaction time (R) & $399(110)$ & $398(94)$ & 0.99 \\
& Auditory reaction time (L) & $364(176)$ & $389(181)$ & 0.53 \\
& Visual reaction time (R) & $428(191)$ & $410(132)$ & 0.82 \\
& Visual reaction time (L) & $459(230)$ & $443(172)$ & 0.80 \\
Ravens matrices & $25.2(5.9)$ & $22.0(6.4)$ & 0.40 \\
Beft & $10.5(6.2)$ & $5.5(4.7)$ & 0.13 \\
& RAVLT depression & $29.7(13.7)$ & $44.2(12.6)$ & 0.13 \\
& Vocabulary & $42.5(10.9)$ & $22.6(11.0)$ & 0.96 \\
& Phonemic fluency & $24.8(13.6)$ & $12.7(3.2)$ & 0.24 \\
Semantic fluency & $14.1(4.6)$ & $12.7(4.2)$ & 0.25 \\
Digit span (total) & $12.9(3.6)$ & $335(150)$ & 0.76 \\
Auditory reaction time (R) & $385(149)$ & $306(104)$ & 0.018 \\
Auditory reaction time (L) & $393(293)$ & $382(104)$ & 0.16 \\
Visual reaction time (R) & $370(152)$ & $355(104)$ & 0.69 \\
Visual reaction time (L) & $396(176)$ & $23.3(6.8)$ & 0.38 \\
Ravens matrices & $23.0(7.3)$ & $7.0(4.6)$ & 0.72 \\
Beck depression & $7.0(3.3)$ & $26.4(9.9)$ & 1.0 \\
RAVLT & $33.2(12.9)$ & & $0.002^{a}$ \\
\hline$a$
\end{tabular}

${ }^{a} p<0.05$ after Bonferroni adjustment.

ing the same subject before and after the procedure. The observed probabilities are presented as they were observed, however statistical significance was inferred based on a Bonferroni style correction [8]. The results are presented in Table 1.

It is clear from this analysis that for the right-sided operations there were no changes on any of the variables post-operatively. The two variables that approached significance were the tendency for the subjects to improve their performance on the total number of words learned on the five trials of the RAVLT, and a moderate improvement in mood as measured by the Beck Depression Inventory which moved from borderline mood disturbance pre-operatively, to no mood disturbance post-operatively. This change in mood did not correlate with the change in performance on the memory measures (all $p>0.05$ ).

For the left lesioned group, the only measure which remained significant after a Bonferroni-type correction was the deterioration in total number of words learned on the five trials of the RAVLT.

A more in depth analysis of the patterns of performance of the two groups on the list learning test is presented in Table 2. It is possible to examine the pattern of performance on the RAVLT in more detail by comparisons within the profile of the performances as proposed by Geffen and Forrester [6]. This ap- proach analyses the acquisition, proactive and retroactive interference effects as well as the recognition and intrusion errors of the subjects.

\subsection{Acquisition}

Difference in performance across the acquisition trials (i.e., Trials $1-5$ on the RAVLT) were examined for each side of lesion using a 2 (pre-versus post-operative) by 5 (Trials 1-5) analysis of variance. The right sided lesions indicated no significant effect for time (i.e., preversus post-operative $[F(1,5)=10.57, p=0.023]$, trials on the RAVLT $[F(4,20)=3.7, p=0.021]$, nor a significant interaction $[F(4,20)=2.18, p=0.108]$ after correction for multiple comparisons.

In contrast the performance of the left-lesioned subjects indicated a significant effect for time (i.e., pre versus post operative $[F(1,12)=153.78, p<0.0005]$ and for trials on the RAVLT $[F(4,48)=12.29$, $p<0.0005]$, and a significant interaction $[F(4,48)=$ 14.76, $p<0.0005]$. This result indicates that the subjects improved as a function of trials, significantly deteriorated from the pre- to the post-operative state and did so in a manner which was different after the operation as compared to that before. Inspection of the shape of the function presented in Table 2 indicates that the subjects with left sided lesions demonstrate 
Table 2

Patterns of performance of the subjects on the Rey Auditory Verbal Learning Test (RAVLT) as a function of side of procedure and relative to operation

\begin{tabular}{|c|c|c|c|c|}
\hline \multirow[t]{2}{*}{ Trial } & \multicolumn{2}{|c|}{ Right side $(n=6)$} & \multicolumn{2}{|c|}{ Left side $(n=13)$} \\
\hline & $\begin{array}{l}\text { Pre-operative } \\
\text { Mean (SEM) }\end{array}$ & $\begin{array}{c}\text { Post-operative } \\
\text { Mean (SEM) }\end{array}$ & $\begin{array}{l}\text { Pre-operative } \\
\text { Mean (SEM) }\end{array}$ & $\begin{array}{c}\text { Post-operative } \\
\text { Mean (SEM) }\end{array}$ \\
\hline 1 & $3.8(1.4)$ & $4.5(1.0)$ & $4.0(0.6)$ & $3.0(0.5)$ \\
\hline 2 & $5.7(1.2)$ & $6.8(0.8)$ & $5.8(0.7)$ & $4.7(0.6)$ \\
\hline 3 & $6.3(1.0)$ & $6.7(1.2)$ & $6.6(0.7)$ & $5.6(0.6)$ \\
\hline 4 & $6.8(1.6)$ & $7.3(1.0)$ & $8.1(0.9)$ & $6.5(0.7)$ \\
\hline 5 & $7.0(1.4)$ & $9.0(1.4)$ & $8.8(0.8)$ & $6.6(0.7)$ \\
\hline List B & $3.7(1.7)$ & $3.0(0.9)$ & $2.4(0.6)$ & $3.4(0.6)$ \\
\hline 6 & $3.7(1.7)$ & $5.5(1.1)$ & $6.9(1.1)$ & $4.7(0.8)$ \\
\hline Recognition & $12.0(0.5)$ & $10.7(1.1)$ & $12.0(0.5)$ & $12.6(0.6)$ \\
\hline Intrusions & $6.5(4.4)$ & $3.2(3.0)$ & $4.1(1.8)$ & $4.1(1.8)$ \\
\hline
\end{tabular}

both attenuation of their verbal learning curve as well as a general depression of the level of the performance from the pre- to the post-operative level.

\subsection{Pro-active interference}

Pro-active interference effects were examined by comparing trial 1 with performance on List $\mathrm{B}$, a previously unseen list which is presented after five trials with List A. The performance was examined for each side of lesion using a 2 (pre-versus post-operative) by 2 (Trial 1, List A versus List B) analysis of variance. The right sided lesions indicated no significant effect for trial $[F(1,5)=3.83, p=0.108]$, list $[F(1,5)=1.0$, $p=0.374]$, or the interaction of these two variables $[F(1,5)=0.04, p=0.856]$.

In contrast the left sided lesions indicated no significant effect for trial $[F(1,9)=2.81, p=0.128]$, or list $[F(1,9)=0.0, p=1.0]$, but a significant interaction effect $[F(1,9)=9.47, p=0.013]$ after the correction. This indicates that before the operation the left-sided lesions indicate a proactive interference effect, which is overcome by the surgery, leading to a release from proactive interference in these subjects post-operatively. This result indicates that the interference effect of the previously presented list does not occur after the surgery, which is consistent with the fact that the information does not impinge as it has already been forgotten.

\subsection{Retroactive interference}

Trials 5 and 6 are compared as a measure of retroactive interference. The performance was examined for each side of lesion using a 2 (pre-versus post-operative) by 2 (Trial 5 versus Trial 6) analysis of variance. The right sided lesions indicated only a significant effect for trial $[F(1,5)=16.91, p=0.009]$, but not for list $[F(1,5)=3.48, p=0.121]$, or the interaction $[F(1,5)=0.01, p=0.927]$.

In contrast the left sided lesions indicated a significant effect for Trial $[F(1,9)=17.23, p=0.002]$, and list $[F(1,9)=17.63, p=0.002]$, but not a significant interaction effect $[F(1,9)=0.29, p=0.606]$. This result indicates that there is a significant deterioration in recall following the intervening list, and following surgery, but the pattern does not differ across the two conditions.

\subsection{Recognition}

Recognition and intrusion performance did not differ across the conditions. Recognition for the right lesioned subjects was the same before as compared to after surgery as assessed using a matched pairs t test $[t(5)=-1.10, p=0.318]$. A similar performance was noted for the left lesioned subjects $[t(9)=0.74$, $p=0.475]$. Intrusions for the right lesioned subjects was the same before as compared to after surgery $[t(5)=-2.10, p=0.089]$. A similar performance was noted for the left lesioned subjects $[t(9)=0.23$, $p=0.823]$.

Unfortunately due to the brevity of the battery of tests it was not possible to comment on the effect of right-sided lesions on non-verbal learning and recall.

None of the pre-operative indicators of motor impairment (Hoehn and Yahr staging, motor score from the UPDRS) nor the alteration in motor function following the procedure was correlated with the level of memory impairment (all $p>0.05$ ). This indicates that the memory effect is independent of the effect of the surgery on motor function. 


\section{Discussion}

The patterns of performance on the verbal learning tests revealed by this study indicate that the unilateral application of PVP to the left side results in a moderate level of verbal memory loss. The result is specific as right-sided lesions not only do not indicate decrease in performance after operation, but actually show a non-significant trend towards improvement.

This finding throws some light on the previous investigations in the area and support the findings of $\mathrm{Ri}$ ordan et al. [16] and Lang [10] of a deficit in verbal memory following the left unilateral procedure. The effects of Soukup et al. [17] and Cullum [4] are also possibly explained by this result in that their much larger sample of right sided lesions, may have masked the deficit in the left lesioned group.

The interesting thing about this result is that such a specific finding has emerged from what has traditionally been considered to be a cognitively silent area. The data from this study indicate that subjects given a left PVP indicate attenuation of their verbal learning curve and a general depression of their level of acquisition of the 15-word list. They also feature a release from pro-active interference following the procedure and do not reveal deterioration in recognition memory or level of intrusion.

Whilst there has been some suggestion in the literature that PVP may be associated with striato-frontal compromise (e.g., $[10,15])$, the absence of a differential frontal compromise in these subjects as measured by the lack of effect of the surgery on the small sample of other cognitive tests assessed in this study, seems to suggest that the possible effect of the procedure may be specific to the memory circuitry. Such a suggestion has previously been made in studies of pallidal lesions to the memory circuitry of the cat [5]. The pattern of performance is consistent with the notion of the classic amnesic syndrome (e.g., [1, 14]) possibly as a consequence of the lesion affecting the relationship with the thalamus resulting in a 'thalamic' memory deficit (e.g., [7]). Nonetheless the possibility of striato-frontal compromise cannot be excluded on the basis of this select data collection, and considerably more investigation into this fascinating issue is indicated.

The post-operative improvement in motor function and reduction in dyskinesias in our patients is consistent with other reports on unilateral PVP [2, 9]. Post-operative neuroimaging confirmed correct lesion placement within the ventral pallidum. Although there was no evidence of haemorrhage within the parasaggital needle tracts from the frontal cortex to the pallidum, it can not be ruled out that damage as a result of electrodes passing through this region contributed to the post-operative verbal memory deficit.

The patterns of performance on the verbal learning tests revealed by this study indicate that left unilateral PVP results in a moderate level of verbal memory loss. The lack of change in performance in patients with right sided lesions suggests that the deficit is specific to the left side. Clearly the results of this investigation raise concern about the cognitive effects of the procedure, and the clinical significance of the results. In the few patients that we have reassessed at 12 months after the operation the impairment of memory seems to persist, and in three of the five patients the family complained of memory-related problems.

\section{References}

[1] A.D. Baddeley, Human memory: Theory and practice, Lawrence Erlbaum, Hove, UK, 1990.

[2] M.S. Baron, J.L. Vitek, R.A.E. Bakay, J. Green, Y. Kaneoke, T. Hashimoto, R.S. Turner, J.L. Woodard, S.A. Cole, W.M. McDonald and M.R. DeLong, Treatment of advanced Parkinson's disease by posterior GPi pallidotomy: 1 year results of a pilot study, Annals of Neurology 40 (1996), 355-366.

[3] M.S. Baron, J.L. Vitek, R.A.E. Bakay, J. Green, Y. Kaneoke, T. Hashimoto, R.S. Turner, J.L. Woodard, S.A. Cole, W.M. McDonald and M.R. DeLong, Treatment of advanced Parkinson's disease by posterior GPi pallidotomy: 1 year results of a pilot study, Annals of Neurology 41 (1997), 835-836.

[4] C.M. Cullum, L.H. Lacritz, A.B. Frol, K.K. Brewer, C. Ciller and R. Dewey, Effects of pallidotomy on cognitive function in Parkinson's disease (abstract), Journal of the International Neuropsychological Society 3 (1997), 61.

[5] L.M. Gambaryan and Z.S. Sarkisyan, Role of the globus pallidus in the mechanisms of memory, Neuroscience and Behavioral Physiology 13 (1983), 470-475.

[6] G.M. Geffen and G. Forrester, Verbal memory functions across adulthood and after moderate to severe closed head injury, in: Proceedings of the Fourteenth Annual Brain Impairment Conference, V. Anderson and M. Bailey, eds, Australian Society for the Study of Brain Impairment, Melbourne, Australia, 1989, pp. 277-284.

[7] N.R. Graff-Radford, H. Damasio and T. Yamada, Nonhaemorrhagic thalamic infarction, Brain 108 (1985), 485-516.

[8] H.C. Howell, Statistical methods for Psychology, Duxbury Press, Belmont CA, 1992.

[9] L.V. Laitinen, A.T. Bergenheim, M.I. Hariz, Leksell's posteroventral pallidotomy in the treatment of Parkinson's disease, Journal of Neurosurgery 76 (1992), 53-61.

[10] A.E. Lang, A.M. Lozano, R. Tasker, J. Duff, J.A. Saint Cyr and L.L. Trepanier, Neuropsychological and behavioral changes and weight gain after medial pallidotomy, Annals of Neurology 41 (1997), 834-835.

[11] J.W. Langston, H. Widner, C. Goetz, D. Brooks, S. Fahn, T. Freeman and R. Watts, Core Assessment Program for In- 
tracerebral Transplantation, Movement Disorders 7 (1992), 2-13.

[12] M.D. Lezak, Neuropsychological assessment, Oxford, New York, 1995.

[13] J. O'Sullivan, R.F. Peppard, P.M. McNeill, S.F. Crowe and M. Hoffman, Unilateral pallidotomy for Parkinson's disease: preliminary results, Journal of Clinical Neuroscience 4 (1997), 404

[14] A.J. Parkin, Memory: Phenomena, experiment and theory, Blackwell, Oxford, 1993.

[15] A. Partiot, M. Verin, B. Pillon, C. Teixeira-Ferreira, Y. Agid and B. Dubois, Delayed response tasks in basal ganglia lesions in man: further evidence for a striato-frontal cooperation in behavioural adaptation, Neuropsychologia 34 (1996), 709-721.
[16] H.J. Riordan, L. Flashman, K. Carroll and D. Roberts, Neurocognitive and psychosocial correlates of ventroposterolateral pallidotomy surgery in Parkinson's disease, Neurosurgery Focus 2 (1997), 1-9.

[17] V.M. Soukup, F. Ingram, M.C. Schiess, J.G. Bonnen, H.J. Nauta and J.R. Calverley, Cognitive sequelae of unilateral posteroventral pallidotomy, Archives of Neurology 54 (1997), 947-950.

[18] L.R. Squire, Mechanisms of memory, Science 232 (1986), 1612-1619.

[19] A.E. Taylor and J.A. Saint Cyr, The neuropsychology of Parkinson's disease, Brain and Cognition 28 (1995), 281296. 


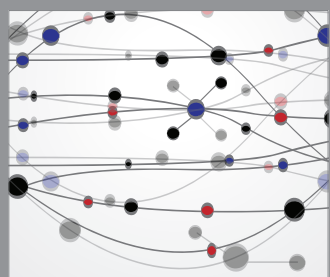

The Scientific World Journal
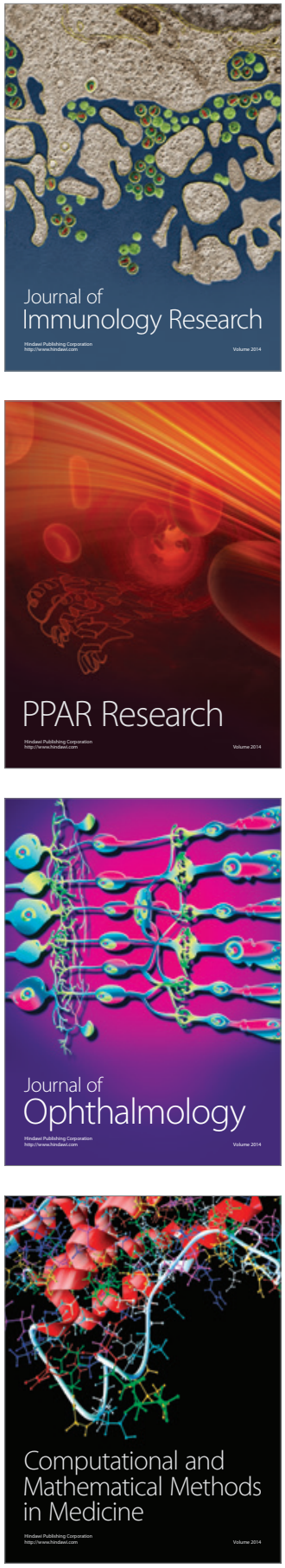

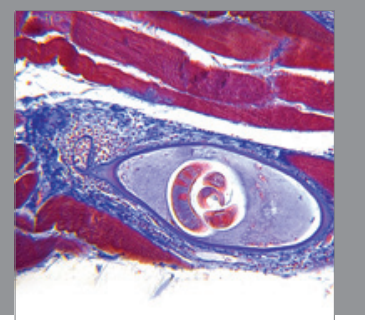

Gastroenterology

Research and Practice
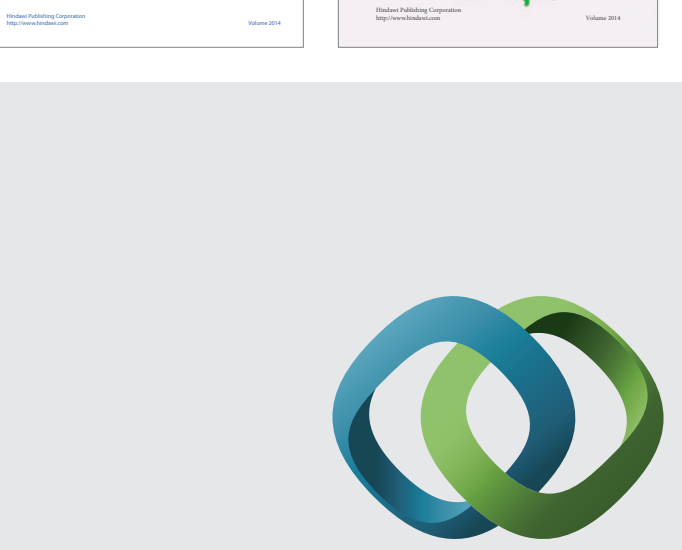

\section{Hindawi}

Submit your manuscripts at

http://www.hindawi.com
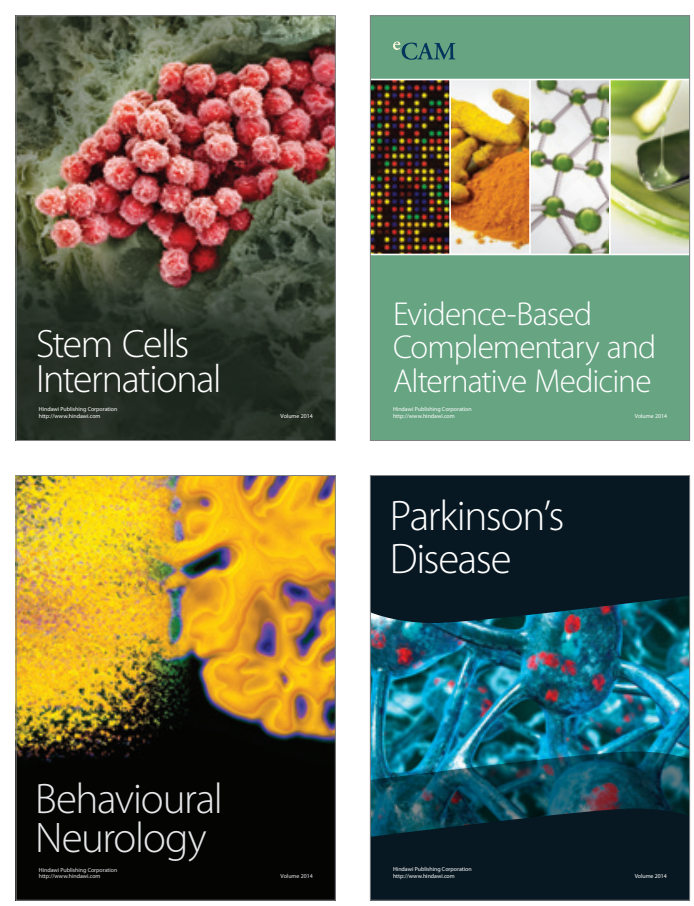

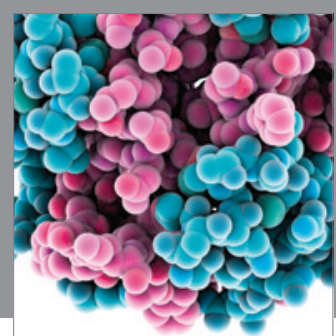

Journal of
Diabetes Research

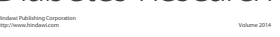

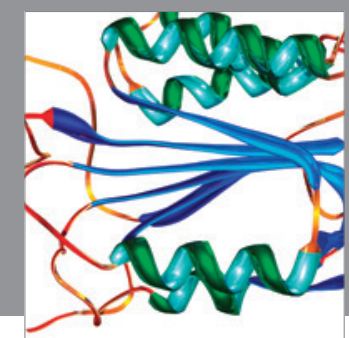

Disease Markers
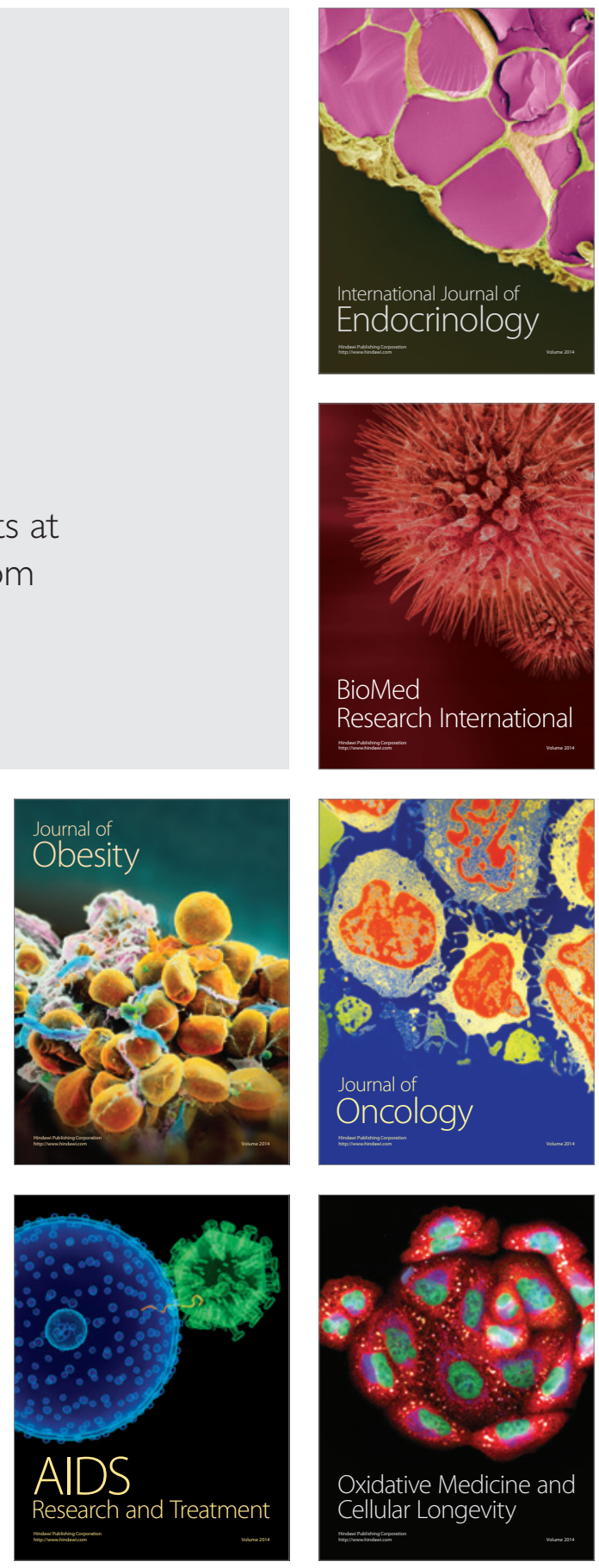